\title{
Smart polymeric nanocarriers for small nucleic acid delivery
}

\author{
Kanjiro Miyata ${ }^{1,2, *}$ \\ 'Department of Materials Engineering, Graduate School of Engineering, The University of Tokyo, Tokyo, Japan; \\ ${ }^{2}$ Center for Disease Biology and Integrative Medicine, Graduate School of Medicine, The University of Tokyo, Tokyo, Japan.
}

\begin{abstract}
Summary In recent decades, nucleic acid therapeutics have been attracting much attention because of their potential as treatments for various diseases that require high target specificity. Nevertheless, the number of approved nucleic acid drugs is very limited due to substantially poor bioavailability, thereby generating the need for nucleic acid delivery carriers. A variety of delivery formulations have been reported so far. This review particularly describes polymer-based delivery carriers for systemic administration, highlighting the potential of biological environment-responsive chemistry for overcoming the biological barriers to nucleic acid delivery.
\end{abstract}

Keywords: Drug delivery, antisense oligonucleotide, small interfering RNA (siRNA), block copolymer, polyion complex (PIC)

\section{Introduction}

Over recent decades, small nucleic acids, such as antisense oligonucleotides (ASO) and small interfering RNA (siRNA), have been extensively highlighted as potential drugs for treatment of various intractable diseases, including cancer (1-3). This is because they can regulate the expression of disease-related genes with high target sequence specificity. However, the arrival rate of exogenous nucleic acids from the injected site to the target cell cytoplasm or nucleus, i.e., bioavailability, is substantially low, mainly due to their high susceptibility to enzymatic degradation, and negatively charged macromolecular structures that impede passive diffusion into the cytoplasm across the cell membrane. These obstacles have hampered the translation of nucleic acid-based drugs into pharmaceutical products.

To overcome the poor bioavailability of nucleic acids, various methodologies have been explored. Chemical modifications of the nucleotide backbone are one of the most typical approaches for stabilization

Released online in J-STAGE as advance publication October 17, 2016.

*Address correspondence to:

Dr. Kanjiro Miyata, Department of Materials Engineering, Graduate School of Engineering, The University of Tokyo, 7-3-1 Hongo, Bunkyo-ku, Tokyo 113-8656, Japan.

E-mail:miyata@bmw.t.u-tokyo.ac.jp of nucleic acids in biological fluids with reduced immune responses (2). Indeed, the clinically approved ASOs, fomivirsen and mipomersen, are composed of a phosphorothioate backbone, and the approved RNA aptamer, pegaptanib, contains 2'-F and 2'-O-methyl modifications (2). These backbone modifications not only protect nucleic acids from enzymatic degradation, but also affect their biodistribution and cellular uptake profiles. For instance, phosphorothioate-modified ASOs are reported to bind to plasma proteins in the bloodstream, leading to enhanced accumulation in the liver (4).

A more direct approach for improving the biodistribution and cellular uptake profiles of nucleic acids is to combine them with delivery molecules. Of these, the simplest formulation is a nucleic acid conjugated to a ligand molecule that can bind to a specific molecule (or receptor) on the target cell surface (Figure 1A) (5). One of the most successful examples is siRNA conjugated with $N$-acetyl-D-galactosamine (GalNAc), which targets the asialoglycoprotein receptor (ASGPR) expressed on the hepatocyte surface (6). This formulation is undergoing a phase III clinical trial (3). Another major formulation is poly(ethylene glycol) (PEG)-coated (PEGylated) nanoparticles (Figure 1B), which can be fabricated by the self-assembly of negatively charged nucleic acids and cationic lipids or polycations bearing PEG chains $(7,8)$. In this formulation, the nanoparticle core can protect nucleic acid payloads from enzymatic degradation and the 




Figure 1. Examples of nucleic acid delivery formulations. (A) Ligand-siRNA conjugate and (B) siRNA-loaded PEGylated lipid nanoparticle.

PEG shell can suppress nonspecific protein adsorption through the steric repulsion effect, permitting the longevity of nucleic acids in harsh in vivo conditions. Notably, PEGylated nanoparticles with a size of several tens of nanometers can preferentially accumulate in solid tumors through leaky tumor vasculature, which is termed the enhanced permeability and retention (EPR) effect $(9,10)$, following systemic administration. Indeed, several formulations of anticancer drug-loaded PEGylated nanoparticles are clinically approved or undergoing clinical trials (11-13). It should be also noted that targeting ligand molecules can be installed onto the surface (or the PEG terminus) of PEGylated nanoparticles, generating a multivalent binding effect for enhanced target recognition $(8,11)$.

Among the structural components of PEGylated nanoparticles, block copolymers comprising PEG and polycation segments are highly appealing because they can be readily functionalized by chemical modification to create multifunctional polymeric nanocarriers $(7,14)$. In particular, biological environment-responsive chemistries, including redox potential- and $\mathrm{pH}$-sensitive linkages, enable block copolymers to generate "smart" polymeric nanocarriers that can exhibit the desired delivery functions, such as reversible stability, targeting ability, and endosome-escaping ability, in response to a specific biological environment or signal. The present review describes the design of smart polymeric nanocarriers and recent progress toward systemic nucleic acid delivery mainly to solid tumors.

\section{Biological barriers in nucleic acid delivery}

Prior to the structural design of block copolymers, this chapter describes the biological barriers to nucleic acid delivery, as well as the prerequisites for nucleic acid-loaded nanocarriers. As illustrated in Figure 2, nucleic acid delivery is divided into two major stages: to the extracellular milieu, and into the intracellular compartment. In the case of systemic administration, nanocarriers need to stably circulate in the bloodstream without disintegration or distribution into non-target

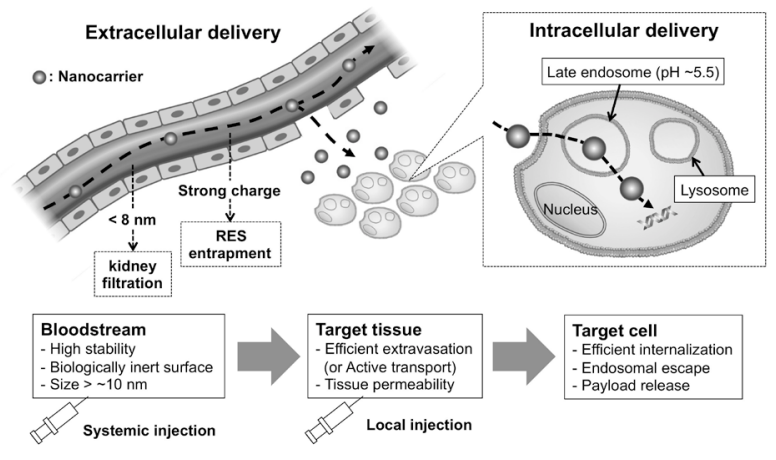

Figure 2. Schematic illustration of nucleic acid delivery and its biological barriers.

organs/tissues. It is known that relatively small macromolecules (or nanocarriers) with a molecular weight (MW) of less than $\sim 40,000$ (or a diameter of less than $\sim 8 \mathrm{~nm}$ ), e.g., naked ASO and siRNA, are rapidly cleared from the bloodstream via renal excretion $(15,16)$. On the other hand, strongly charged larger macromolecules (or nanoparticles) are more likely to be entrapped by the reticuloendothelial system (RES) via nonspecific protein adsorption, leading to their clearance from the blood (7). Thus, nanocarriers ideally have a size of over $\sim 10 \mathrm{~nm}$ with a nonionic and hydrophilic (or biologically inert) surface, i.e., a PEG shell, to maintain longevity in the bloodstream.

At the target organ/tissue, nanocarriers need to extravasate from the bloodstream. In the case of solid tumors (or inflammatory tissues), the vascular pores are relatively large with sizes of $>100 \mathrm{~nm}$, allowing nanocarriers with a size of $<100 \mathrm{~nm}$ to extravasate toward the tumor tissue. This is known as the EPR effect $(9,10)$. Meanwhile, the size of vascular pores in healthy organs/tissues, except for the liver and spleen, is less than $10 \mathrm{~nm}$, and thus, active transport mechanisms, e.g., transcytosis, may be required for nanocarriers targeting healthy organs/tissues with smaller vascular pores. After extravasation or in the case of local administration, nanocarriers need to deeply permeate the target tissue to access a wide range of target cells. Tumor tissue permeability of nanocarriers is substantially affected by tumor heterogeneity or histological characteristics $(17,18)$. When a series of anticancer drug-loaded nanocarriers with varying sizes of $30,50,70$, and $100 \mathrm{~nm}$ were intravenously administered into subcutaneous tumorbearing mice, their tumor accumulation profiles were dramatically altered according to the tumor model. In the case of a hypervascular colon (C26) tumor model, all the nanocarriers showed similar, efficient tumor accumulation ( $10 \%$ dose/g tissue) (Figure 3A) (18). In contrast, in the case of a thick fibrotic, hypovascular pancreatic (BXPC3) tumor model, the smaller nanocarriers more efficiently accumulated in the tumor tissue, compared with the larger ones, associated with 
(A) Subcutaneous colon tumor model with C26

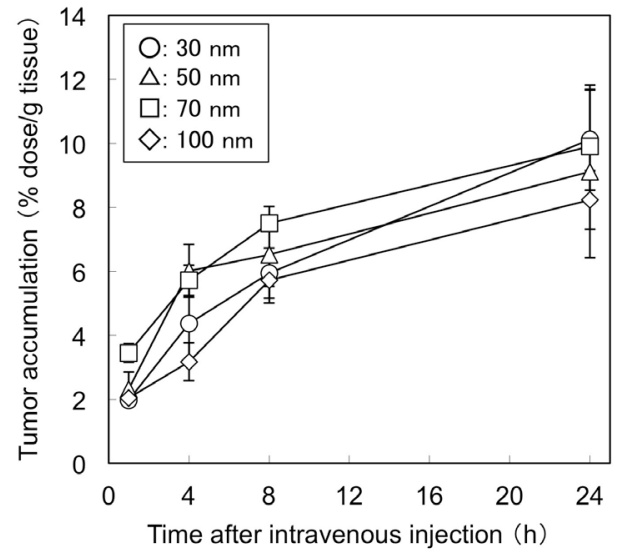

(B) Subcutaneous pancreatic tumor model with BxPC3

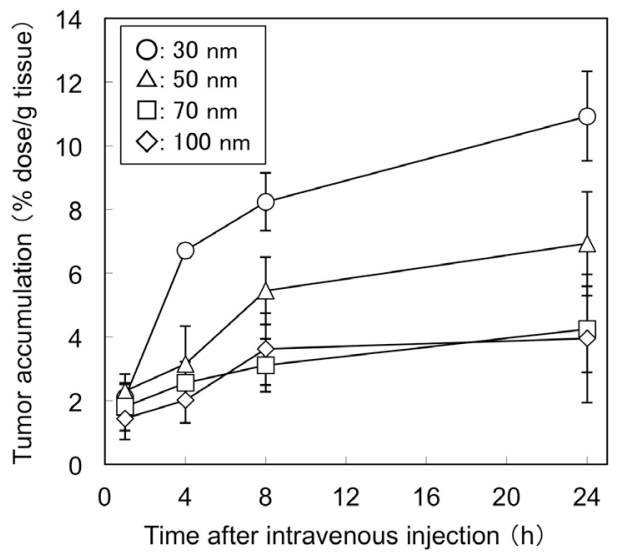

Figure 3. Tumor accumulation profiles of anticancer drug-loaded polymeric micelles after systemic administration. The polymeric micelles were prepared from a platinum anticancer drug, 1,2-diaminocyclohexane-platinum(II), and a mixture of PEGpoly(glutamic acid)/homo-poly(glutamic acid) to produce varying hydrodynamic diameters. A subcutaneous tumor model was developed using a colon cancer cell line, C26 (A), and a pancreatic cancer cell line, BxPC3 (B). These results were originally reported in reference 18 .

an apparent size threshold of $\sim 50 \mathrm{~nm}$ (Figure 3B) (18). These results strongly suggest that smaller nanocarriers with a size of $<50 \mathrm{~nm}$ are more suitable for deep permeation within heterogenous tumor environments.

After reaching the target cells, nanocarriers need to migrate to the cytoplasm (or nucleus) by overcoming cellular barriers, namely the cytoplasmic membrane and the endosomal membrane (and nuclear membrane if the target site is the nucleus). Nanocarriers are generally uptaken by cells via adsorptive endocytosis $(4,14)$. Thus, stronger binding affinity to the target cellular surface is likely to result in more efficient cellular internalization of nanocarriers. However, a stronger binding ability conflicts with the biological inertness derived from the PEG shell that allows for avoiding RES entrapment as well as nonspecific protein adsorption. To overcome this conflicting issue, the so-called PEG dilemma, the PEG shell can be further modified with a targeting ligand molecule for more specific binding, as aforementioned. Otherwise, the PEG shell can be designed to disintegrate from the nanocarrier by utilizing cleavable linkages at the target site. After endocytosis, nanocarriers are generally subjected to endosomal sorting toward lysosomal digestion, and thus, they must escape from the endosomal compartment into the cytoplasm, where the nucleic acid payloads must be released from the nanocarrier to access the gene silencing machinery, hybridize with the complementary messenger RNA, or form the RNA-induced silencing complex (RISC).

\section{Design of block copolymers for nucleic acid delivery}

This chapter describes the design of block copolymers for construction of smart polymeric nanocarriers, especially for systemic siRNA delivery to solid tumors.
The first section focuses on the basic properties of polymeric nanocarriers prepared with PEG-polycation block copolymers. The following sections highlight the functionalization of block copolymers to improve the delivery of polymeric nanocarriers.

\subsection{Basics of block copolymer self-assembly}

When PEG-polycation block copolymers are mixed with nucleic acids in aqueous solutions, they can spontaneously assemble into polyion complexes (PICs) through charge neutralization. This PIC formation can be explained by a two-step assembly process, as illustrated in Figure 4A (19). The primary step forms a minimal charge-neutralized polyion pair between a PEG-polycation and a nucleic acid, termed a unit PIC (uPIC). Above a critical association concentration (CAC), uPICs undergo secondary assembly into micellar PICs (mPICs). This two-step self-assembly is clearly demonstrated when single-strand RNA (ssRNA) (21-mer) is combined with PEG-poly(Llysine) (PEG-PLys) (MW of PEG $\left(\mathrm{MW}_{\mathrm{PEG}}\right): 12,000$, degree of polymerization of PLys $\left(\mathrm{DP}_{\mathrm{PLys}}\right)$ : 40) (Figure 4B) (20). At lower concentrations $(<0.01 \mathrm{mg} / \mathrm{mL})$, the formation of small PICs with a hydrodynamic diameter $\left(D_{H}\right)$ of $\sim 10 \mathrm{~nm}$ is clearly observed, corresponding to the primary assembly step, i.e., uPICs. In contrast, the $D_{H}$ value is obviously increased at concentrations greater than $0.01 \mathrm{mg} / \mathrm{mL}$, ultimately reaching $\sim 40 \mathrm{~nm}$. The transmission electron microscopic (TEM) image of PIC samples prepared at higher concentration displays the spherical nanoparticles (Figure 4C). These results demonstrate the formation of micelles from uPICs through the secondary assembly step. Meanwhile, a large difference is observed for double-strand RNA (21-mer/21-mer), i.e., siRNA, which maintains the uPIC structure without progressing to secondary 
(A)

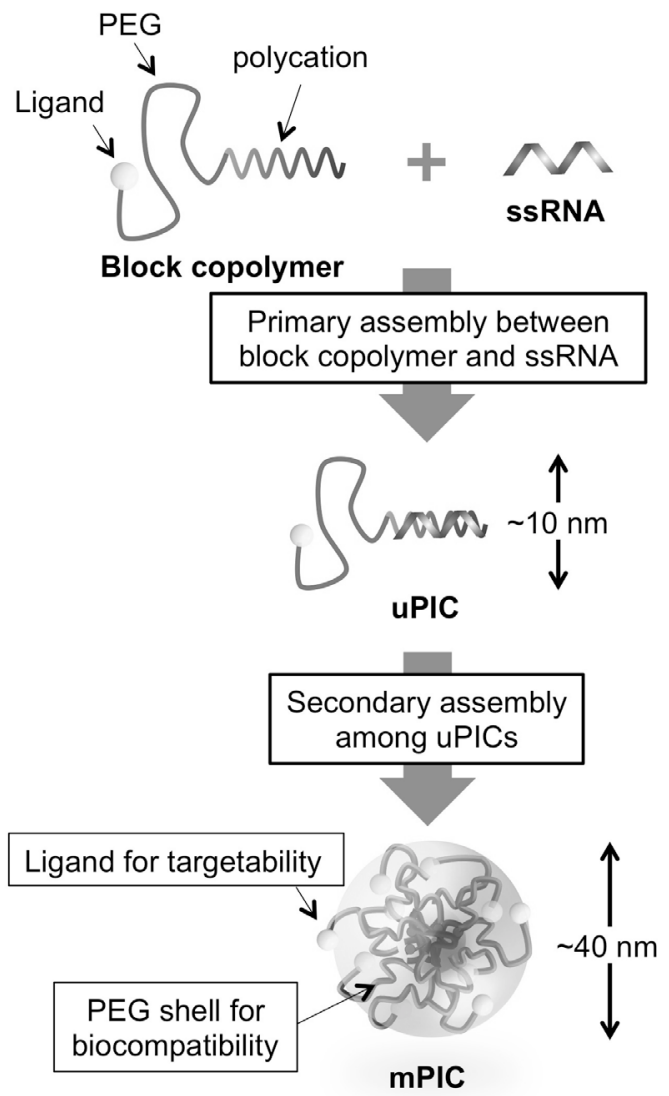

(B)

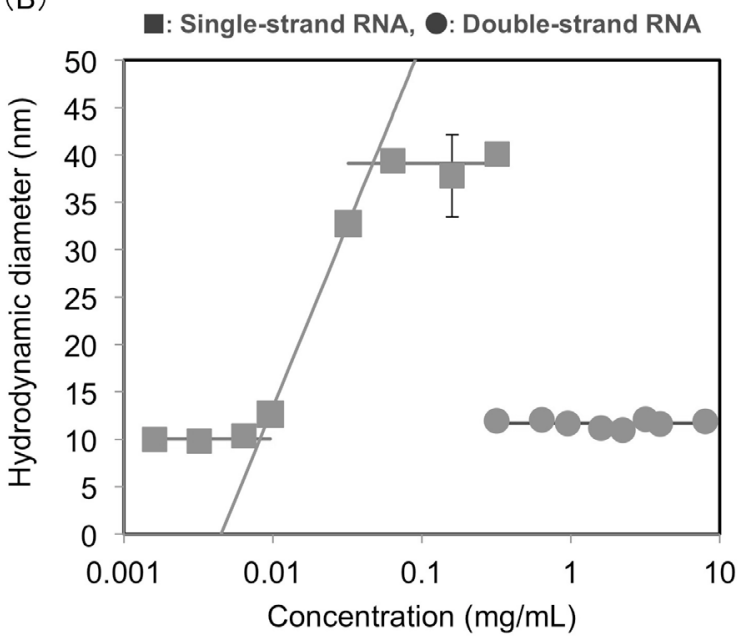

(C)

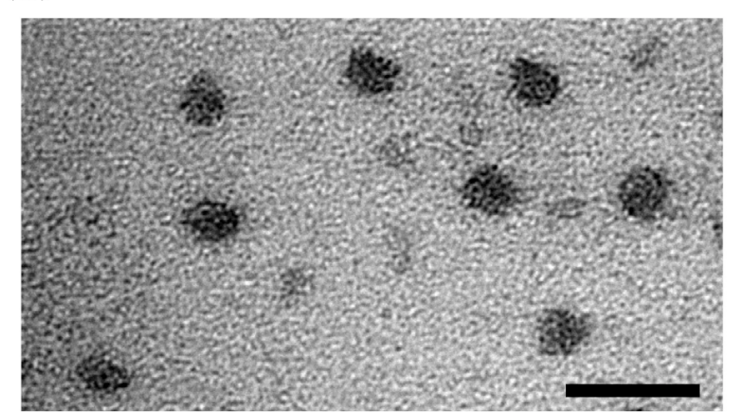

Figure 4. PIC formation between PEG-polycations and nucleic acids. (A) Schematic illustration of the two-step selfassembly from PEG-polycation and small nucleic acid. (B) Size of PIC samples prepared from PEG-PLys and ssRNA or siRNA at different concentrations. The hydrodynamic diameter was determined by fluorescence correlation spectroscopic analysis using fluorescently labeled RNA. (C) TEM image of the PIC sample prepared from PEG-PLys and ssRNA at a concentration of $\sim 0.3$ $\mathrm{mg} / \mathrm{mL}$. Scale bar indicates $50 \mathrm{~nm}$. These results were originally reported in reference 20 .

assembly even at much higher concentrations (Figure 4B). This difference may be explained by the difference in rigidity (or persistence length) between ssRNA and siRNA. siRNA has a persistence length of $60 \mathrm{~nm}$, thereby being considered as having rigid cylindrical architecture. This rigidity potentially compromises the entropy gain that is associated with secondary assembly (or segregated core formation in a micelle), compared with flexible ssRNA with a persistence length of 1 $\mathrm{nm}$, and hence inhibits mPIC formation. These results suggest that additional association forces of siRNAloaded uPICs are crucial for construction of stable siRNA-loaded mPICs.

mPICs have a core-shell architecture, wherein the PEG shell comprises the biologically inert (or biocompatible) surface and can be further modified with ligand molecules for preferential accumulation in target tissues/cells (Figure 4A). The $\mathrm{D}_{\mathrm{H}}$ of oligonucleotideloaded mPICs prepared from PEG-PLys is reported to range from 40 to $80 \mathrm{~nm}$ with a narrow size-distribution (polydispersity index in dynamic light scattering, $\mu_{2} /$ $\left.\Gamma^{2}<0.1\right)(21,22)$. The smaller mPICs were constructed using PEG-PLys with shorter PLys segments, e.g., $\mathrm{DP}_{\mathrm{PLys}}=\sim 20$ and $\sim 40$, whereas PEG-PLys with a longer
PLys segment $\left(\mathrm{DP}_{\mathrm{PLys}}=\sim 80\right)$ generated the larger mPICs $(20,22)$. Thus, the smaller mPICs are potentially highly efficient for permeation within solid tumor tissues, in contrast with lipid nanoparticles with a $D_{H}$ of $\sim 90 \mathrm{~nm}$ (23).

To fabricate more stable mPICs, the polycation segment in block copolymers can be modified with stabilizing units. In PEG-PLys, the modification of primary amines in the PLys segment with a guanidino group significantly enhances the stability of siRNAloaded mPICs, possibly due to the hydrogen bonding (24). Additionally, hydrophobic groups, such as cholesteryl groups and stearoyl groups, or hydrophobic polymer segments, can be introduced into the side chains or the $\omega$-end of the polycation segment in block copolymers for stabilization of the mPIC through hydrophobic interactions (Figure 5) (25-27). Conversely, hydrophobic modifications can also be applied to nucleic acids instead of the polycation segment; cholesterol-modified siRNA allowed for preparation of more stable mPICs compared with non-modified siRNA (28). As another stabilizing approach, mPICs can be coated with an additional outer layer, such as silica gel, which is readily prepared by simply mixing mPICs with silicate ions (29). 




$\mathrm{X}$ : Stabilizing unit

- hydrogen bonding moiety

- hydrophobic moiety

- hydrophobic polymer segment

Figure 5. Modification sites of block copolymers for preparation of stable mPICs.

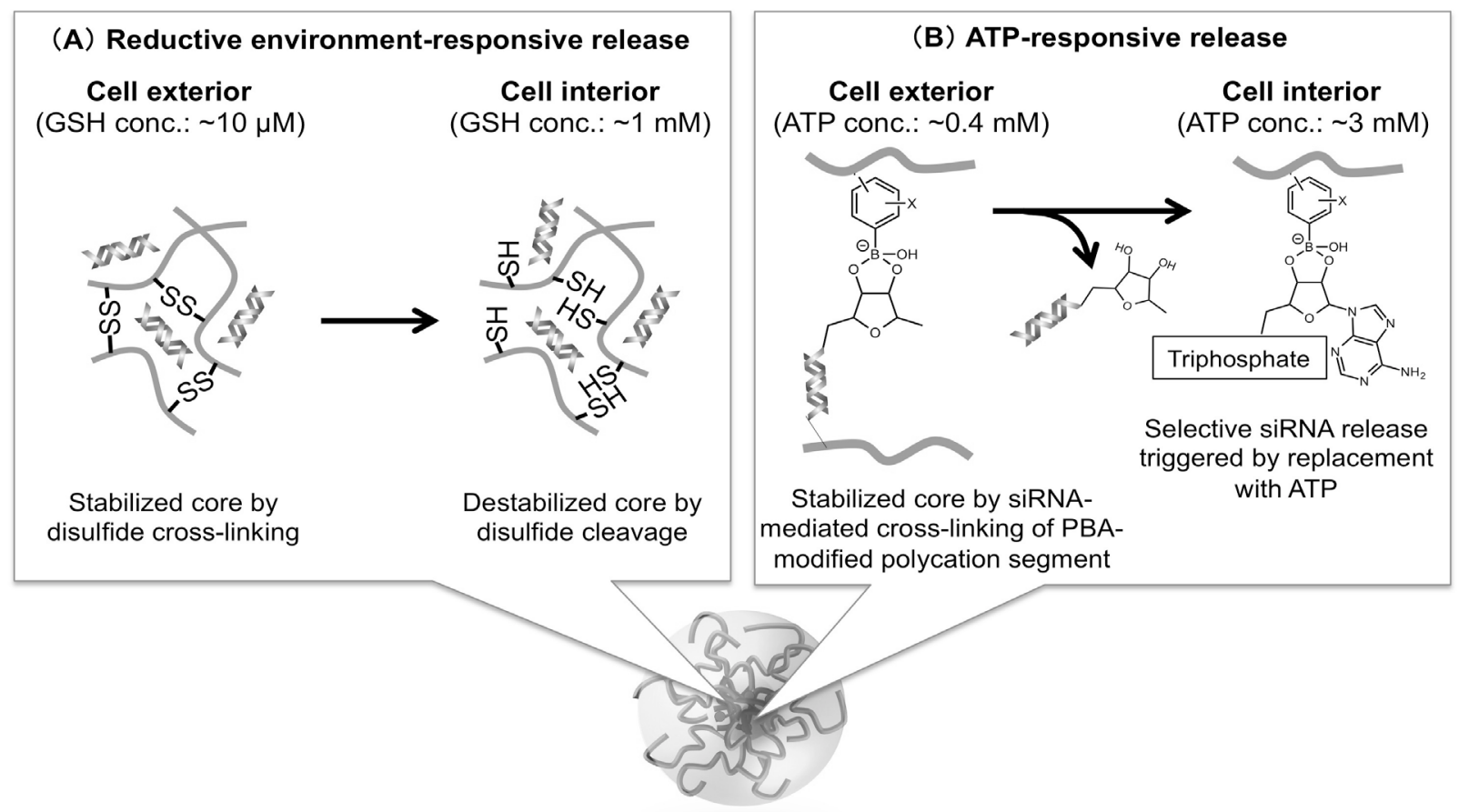

Figure 6. Design of smart polymeric nanocarriers using environment-responsive block copolymers for programmed nucleic acid release. (A) Disulfide cross-linked mPICs that can be destabilized in response to intracellular reductive conditions. (B) PBA-functionalized mPICs where siRNA can be released by replacement with intracellular ATP.

\subsection{Polymer design for reversible stability}

Whereas nanocarriers need to stably encapsulate nucleic acid payloads in extracellular milieu, they must ultimately release the payloads for effective gene silencing at the target site of action. To fulfill this apparently conflicting demand, block copolymers and their polymeric nanocarriers can be functionalized by environment-responsive chemistry for reversible stability or programmed payload release. Among various biological parameters, redox potential and $\mathrm{pH}$ have been most extensively utilized to trigger an environmental response from block copolymers because their levels are drastically altered between the interior and exterior of the cell. The cytoplasm is known to be a reductive environment, as the concentration of reduced glutathione (GSH), which acts as a reductive agent in the body, is three orders of magnitude higher in the cytoplasm compared with the extracellular milieu
(30). Accordingly, reductive environment-responsive disulfide bonds can be utilized for stabilization of mPICs by cross-linking the polycation segments $(24,28,31-36)$. In this way, the disulfide cross-linked mPICs can be stabilized in non-reductive conditions, while they are destabilized through the cleavage of disulfide bonds in reductive conditions, resulting in accelerated payload release in the cytoplasm (Figure 6A). Similarly, the $\mathrm{pH}$ in the late endosome $(\mathrm{pH}$ $\sim 5.5$ ) is known to be significantly lower than $\mathrm{pH}$ in the extracellular milieu (pH 7.4) and the cytoplasm (pH 7.2) (37). Therefore, cross-linking via acid-labile bonds, such as an acetal group (38), is also useful for the fabrication of acidic $\mathrm{pH}$-responsive nanocarriers that can preferentially release payloads within the late endosome.

Although previous studies have reported a certain extent of stabilization from disulfide cross-linking, the stability of siRNA-loaded mPICs in the bloodstream 
still requires improvement, as the blood half-life is reported to be $\sim 10 \mathrm{~min}(33,34)$. This limitation in the longevity of siRNA-loaded mPICs may be due to significant leakage of siRNA payloads from the crosslinked polycation network in the mPIC. This leakage hypothesis is supported by fairly high stability of the mPICs, with cross-links formed between polycation and polyanion segments, allowing for a blood half-life of $\sim 5 \mathrm{~h}(39)$. This fact suggests that direct conjugation of siRNA with the polycation segment via reversible linkages may be a promising strategy for increasing the longevity of mPICs in the bloodstream. To this end, a previous study highlights the characteristic moiety of siRNA, namely the cis-diol in the ribose ring at the $3^{\prime}$ end of siRNA. The cis-diol group can make a reversible ester bond with tetravalent phenylboronic acid (PBA) groups. Accordingly, siRNA with cis-diol groups can act as a cross-linker between PBA-modified polycation segments through phenylboronic ester bonding, thereby generating the siRNA-mediated cross-linked mPICs (Figure 6B). Notably, this phenylboronic ester bond can be replaced with the cis-diol group contained in adenosine triphosphate (ATP) in a concentrationdependent manner (40). Particularly, whereas the mPICs cross-linked with siRNA were stable at low ATP concentrations of $<1 \mathrm{mM}$, high ATP concentrations of $>2 \mathrm{mM}$ dramatically accelerated siRNA release from the mPICs. Considering that the extracellular and intracellular ATP concentrations are $\sim 0.4 \mathrm{mM}$ and $3 \mathrm{mM}$, respectively (41), these siRNA-mediated cross-linked mPICs are expected to selectively release the siRNA payloads in the intracellular compartment (Figure 6B), which is currently under investigation. On the other hand, the siRNA-mediated cross-linked mPICs achieved a blood half-life of $>60 \mathrm{~min}$ (unpublished data), demonstrating the strong potential of direct conjugation of nucleic acids to polycation segments for increasing the longevity of polymeric nanocarriers in the bloodstream.

\subsection{Polymer design for active targeting and facilitating cellular uptake}

After extravasation from the vessel into the target tissue, nanocarriers should ideally enter the target cells efficiently. In this regard, the PEG shell of mPICs significantly suppresses their cellular internalization through the steric repulsion effect (PEG dilemma). One of the most promising approaches to overcome this dilemma is active targeting using ligand molecules. Indeed, a variety of ligand molecules, including GalNAc, can be installed onto the nanocarrier surface, as reviewed elsewhere $(8,42)$. In this way, multiple ligand-installed nanocarriers permit multivalent binding to target cellular surfaces for enhanced target recognition, which is in sharp contrast with the single ligand-mediated monovalent binding. As a representative ligand, transferrin (Tf), which is a glycoprotein with a MW of $\sim 80 \mathrm{kDa}$, has been used as a cancer cell-targeting ligand over several decades (43) and has been clinically tested in the siRNAloaded cyclodextrin nanoparticle (44). It should be noted that the $\mathrm{Tf}$ ligand is also available for active targeting to brain endothelial cells to elicit transcytosis toward the brain parenchyma across the blood-brain barrier (BBB) (45). In detail, the effect of Tf ligand density on nanoparticles was extensively examined for effective transcytosis across the BBB. Interestingly, the optimized ligand density (or avidity) was observed to cause accumulation of nanoparticles in the brain parenchyma, whereas nanoparticles with a higher density of ligands showed stronger binding to the brain endothelial cellular surface.

Peptide molecules that can be designed with a high selectivity are also promising ligand candidates. For example, a short peptide derived from rabies virus glycoprotein (RVG) (29-mer) is reported to enable the transvascular delivery of siRNA to the brain and specifically bind to the acetylcholine receptor overexpressed on the neuronal cell surface (46). In addition, the cyclic RGD (arginine-glycine-aspartic acid) peptide (cRGD) has been extensively tested for active targeting of $\alpha_{v} \beta_{3}$ and $\alpha_{v} \beta_{5}$ integrins overexpressed on the surface of various cancer cells and cancerrelated endothelial cells (47-49). cRGD-installed mPICs are demonstrated to more efficiently accumulate in subcutaneous tumor models, i.e., $\alpha_{v} \beta_{5}$-overexpressing cervical cancer cells (HeLa and SiHa cells) and lung cancer cells (A549 cells), compared with non-ligandinstalled mPICs. Ultimately, the cRGD-installed/siRNAloaded mPICs achieved significant antitumor activity against these tumor models by intravenously delivering therapeutic siRNAs targeted to vascular endothelial growth factor (VEGF) and its type-2 receptor (VEGF-R2) in the HeLa model (Figure 7A) (34), papilloma virusderived oncogene E6/E7 in the SiHa model (Figure 7B) (36), and polo-like kinase 1 (PLK1) in the A549 model (Figure 7C) (26), associated with sequence-specific gene silencing in the tumor tissues.

An additional important feature of ligand-mediated active targeting is the critical effect of the range of ligand motion on multivalent binding. Although multiple ligand-installed mPICs have a large capacity for multivalent binding to the target molecules, a high density of PEG chains reduces their mobility, as well as the range of ligand motion, because of the steric repulsive (or crowding) effect derived from neighboring PEG chains (Figure 8A). This reduction in the PEG mobility and ligand motion makes it difficult to generate effective multivalent binding to target molecules. As an alternative, cocktail PEGylation of nanoparticles with ligand-installed longer PEG and non-ligand-installed shorter PEG demonstrated dramatically enhanced affinity to the target molecule- 
(A)



(B)



(C)

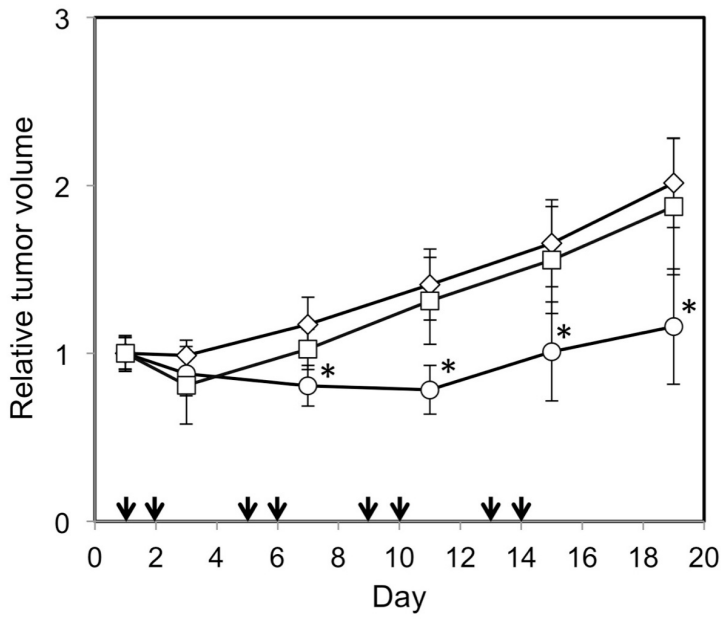

Figure 7. Antitumor activities of cRGD-installed/siRNA-loaded mPICs against subcutaneous cancer models developed from HeLa cells (A), SiHa cells (B), and A549 cells (C). siRNA-loaded mPICs were intravenously injected into the tail vein of tumorbearing mice ( $\sim 25 \mu \mathrm{g}$ siRNA/mouse/shot) on the designated day as indicated by arrows. Open diamond: buffer-treated controls, open square: cRGD-installed/non-therapeutic control siRNA-loaded mPIC-treated mice, open triangle: non-cRGD-installed/therapeutic siRNA-loaded mPIC-treated mice, and open circle: cRGD-installed/therapeutic siRNA-loaded mPIC-treated mice. Therapeutic siRNAs were designed for targeting human VEGF (white arrow) and mouse VEGF-R2 (black arrow) (A), papilloma virus-derived E6/E7 (B), and human PLK1 (C). ${ }^{*} p<0.05$ for buffer-treated control. These results (A), (B), and (C) were originally reported in references 34,36 , and 26 , respectively.

(A) Simple PEGylation with single length



Figure 8. Design of ligand-installed PEGylated nanocarriers for enhanced target recognition. (A) Nanocarrier surface decorated with a single length of PEG, where the range of ligand motion is narrow due to the steric repulsive effect derived from neighboring PEG chains. (B) Enlarged range of ligand motion by cocktail PEGylation using ligand-installed longer PEG, and nonligand-installed shorter PEG as a filler molecule. 
(A)

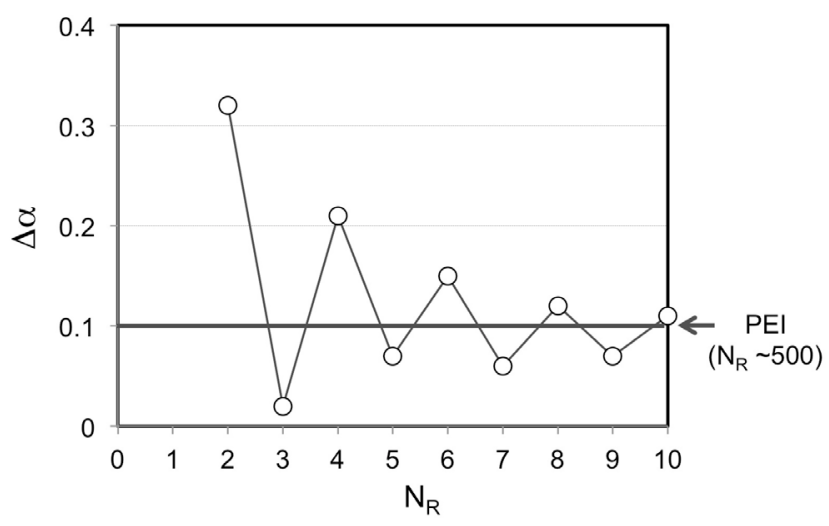

(B)

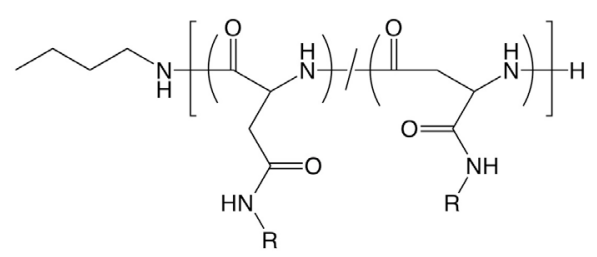

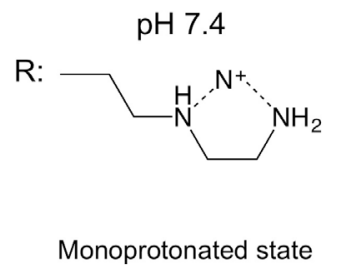

pH 5.5

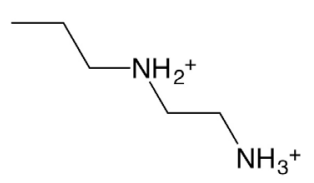

Diprotonated state

(C)

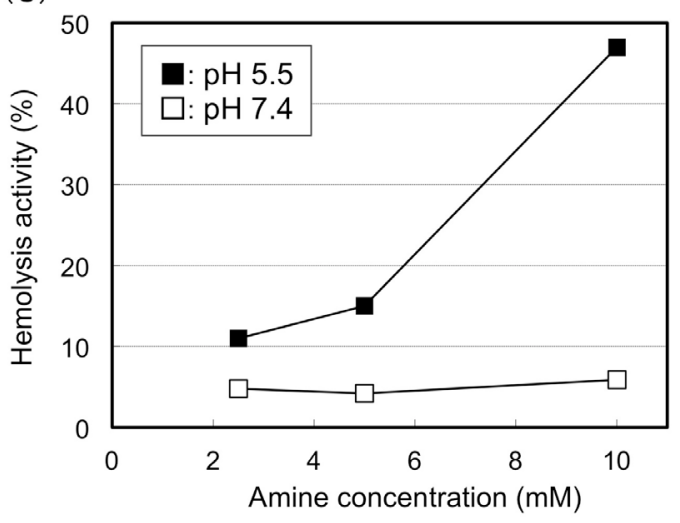

Figure 9. Acidic pH-responsiveness of aminoethylene units and PAsp(DET). (A) Relationship between the repeating number of aminoethylene units $\left(\mathrm{N}_{\mathrm{p}}\right)$ and change in the protonation degree between $\mathrm{pH} 7.4$ and $5.5(\Delta \alpha)$. (B) Protonated structures of PAsp(DET) at $\mathrm{pH} 7.4$ and 5.5. (C) Erythrocyte hemolysis activity of PAsp(DET) determined at $\mathrm{pH} 7.4$ and $\mathrm{pH} 5.5$ plotted against amine concentration. This result was originally reported in reference 59.

immobilized surface, presumably due to the enlarged range of ligand motion (or mobility of PEG chains) produced by the spacer effect of shorter PEG fillers for effective multivalent binding (Figure 8B) (50).

\subsection{Polymer design for endosomal escape}

Once nucleic acid-loaded nanocarriers are endocytosed by cells, they generally undergo endosomal sorting and ultimately lysosomal degradation. Thus, endosomal escape toward the cytoplasm has been a much-discussed issue in nucleic acid delivery. For enabling endosomal escape of nanocarriers, a major approach is endosomal membrane disruption (or destabilization) using membrane-disrupting polycations or peptides. One of the most widely used polycations is polyethylenimine (PEI). PEI contains low pKa amines, which can protonate in response to the endosomal acidic $\mathrm{pH}$ of $\sim 5.5$ (51). This amine protonation is hypothesized to induce the influx of protons and chloride ions into the endosomal compartment, resulting in increased osmotic pressure and ultimately endosome disruption. This is termed the proton sponge effect $(52,53)$. Concurrently, amine protonation provides PEI with greater cationic charge, allowing for stronger binding to the negatively charged endosomal membrane and facilitating destabilization $(14,53)$.

On the other hand, the considerable cytotoxicity of PEI is always a matter of debate around its pharmaceutical application, and thus, many researchers have focused on reducing its cytotoxicity. Generally, the cytotoxicity of polycations is elicited by cationic charges that allow for nonspecific binding to the cytoplasmic and/or mitochondrial membrane. Therefore, more cationic charges/higher cationic charge densities result in greater cytotoxicity $(14,54,55)$. A typical approach to reduce cytotoxicity is the preparation of biodegradable polycations via intracellularly cleavable bonds, such as disulfide bonds and acid-labile bonds (56,57). Low toxicity oligoethylenimines with $\mathrm{MW}$ of $\sim 2 \mathrm{kDa}$ have been conjugated with each other via acid-labile imine linkers for preparation of biodegradable PEI, resulting in significantly reduced cytotoxicity associated with a high level of transfection efficiency (56). Another approach for preparation of low toxic endosomedisrupting polycations is the spatial rearrangement of cationic moieties on the polycation segment for controlled protonation behavior (or cationic charge density). The protonation behavior of the aminoethylene unit, $-\mathrm{NHCH}_{2} \mathrm{CH}_{2}$, which is the repeating unit of PEI, 
(A)

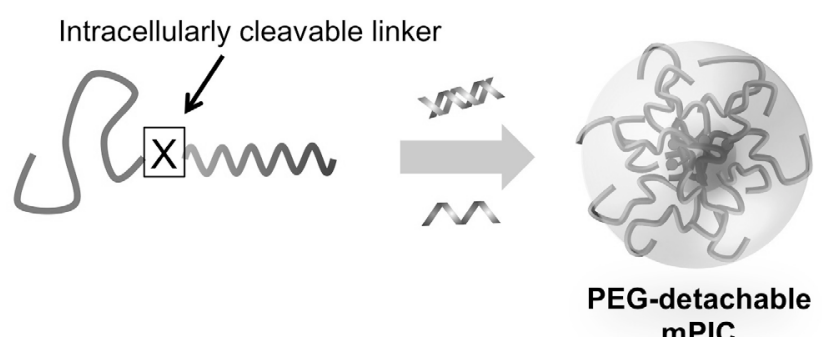
mPIC



(B)

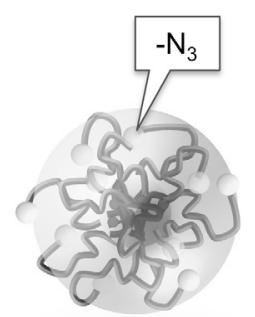

Azide-functionalized MPIC

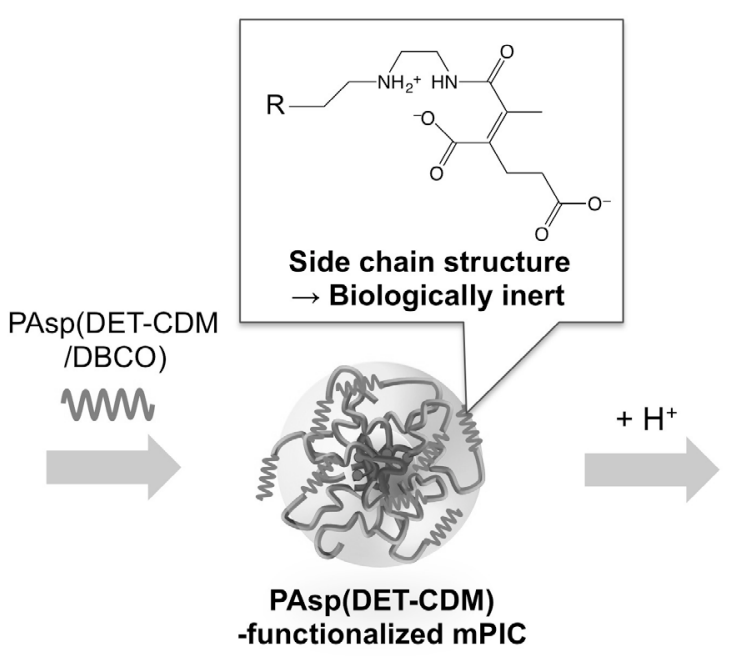

Figure 10. Design of smart polymeric nanocarriers for selective membrane disruption within the acidic endosomal compartment. (A) PEG-detachable mPICs prepared from PEG-polycation, where the PEG and the polycation are connected through intracellularly cleavable linkages, such as acid-labile bonds. (B) Surface-functionalized mPICs using the endosome-disrupting polymer that can become active toward the cell membrane after being unmasked at acidic $\mathrm{pH}$.

is substantially altered by the repeat number $\left(\mathrm{N}_{\mathrm{R}}\right)$; increase in $N_{R}$ progressively reduces the change in the protonation degree $(\Delta \alpha)$ of aminoethylene units between extracellular neutral $\mathrm{pH}$ and endosomal acidic $\mathrm{pH}$ with a simple harmonic motion (Figure 9A) (14,58). This finding suggests that the aminoethylene unit at $\mathrm{N}_{R}$ $=2$ possesses higher endosomal $\mathrm{pH}$-responsiveness, compared with the others. When the twice-repeated aminoethylene moieties were introduced into the side chain of polyaspartamide, the obtained polymer, termed PAsp(DET), maintained high $\Delta \alpha(=0.31)$, which is significantly higher than that of PEI with a DP of $\sim 500$ $(\Delta \alpha=\sim 0.1)(14,59)$. In detail, the protonation state of side chains in PAsp(DET) is the monoprotonated state with a relatively low cationic charge density at neutral $\mathrm{pH}$, which is distinctly converted to the diprotonated state at endosomal acidic $\mathrm{pH}$ (Figure 9B). Interestingly, this change in the protonated state of PAsp(DET) was associated with altered membrane disruption (or hemolysis) activity; hemolysis activity was negligible at neutral $\mathrm{pH}$ but dramatically elevated at endosomal acidic pH (Figure 9C), demonstrating acidic $\mathrm{pH}$ responsive membrane disruptive functionality. Indeed, PAsp(DET) was confirmed to exhibit high transfection efficiency with much lower cytotoxicity, compared with PEI (59).

As expected, the PEG shell of mPICs considerably compromises endosomal membrane-disruptivity of polycations. Thus, biological environmentresponsiveness is still required to render the nanocarrier surface biologically inert in the extracellular milieu but membrane-active within the endosome. One reasonable approach is PEG-detachable formulations, wherein the PEG segment and the polycation segment are connected via intracellularly cleavable bonds, e.g., disulfide (-SS-) bonds and acid-labile bonds (Figure $10 \mathrm{~A})(60,61)$. In this way, systemic delivery of VEGF siRNA by mPICs prepared from PEG-SS-PAsp(DET) featuring stearoyl moieties in the PAsp(DET) side chains exhibits significant antitumor activity in a subcutaneous pancreatic (BxPC3) tumor model (25). Another sophisticated approach is the installation of endosome-disrupting polymers onto the mPIC surface (or PEG terminus). In this case, the polycations need additional chemical modifications to mask their cationic charges (or amines). A successful example of aminemasking is the use of amide linkages derived from maleic acid derivatives, including cis-aconitic acid and 
carboxylated dimethyl maleic acid (CDM), which are relatively stable at neutral $\mathrm{pH}$ yet degradable at acidic $\mathrm{pH}$, regenerating the parent amine moiety (Figure 10B) (62-64). The CDM-modified PAsp(DET) (PAsp(DETCDM)) was successfully conjugated to the PEG terminus of mPIC through copper-free click chemistry between a dibenzocyclooctyne (DBCO) group and an azide group (Figure 10B) (35). The demasking of PAsp(DET-CDM) was verified by an increase in zeta-potential of mPICs at acidic $\mathrm{pH}$, presumably due to the regeneration of cationic PAsp(DET) on the mPIC surface. Ultimately, the PAsp(DET-CDM)-installed mPICs achieved fairly high gene silencing activity in cultured cancer cells, compared with non-installed control mPICs.

\section{Conclusion and perspectives}

This review describes the design of polymeric nanocarriers and recent progress regarding their use in small nucleic acid delivery by highlighting methods to overcome biological barriers. To date, many published works have reported excellent therapeutic outcomes in animal disease models. Notably, multifunctional nanocarriers have elicited significant gene silencing mainly in solid tumors as well as the liver, demonstrating their promising therapeutic potential. Nevertheless, there are still limitations or gaps for translation into pharmaceutical products. One of the major reasons for disruption to translation is the limitation of animal disease models, some (or many) of which may not be good surrogates for human patients. Currently, this issue is being actively tackled by many researchers engaged in biology and drug delivery. The establishment of good surrogates can dramatically facilitate the pharmaceutical translation of oligonucleotide therapeutics.

\section{Acknowledgements}

The research described in this review was supported by the Center of Innovation (COI) program of the Japan Science and Technology Agency (JST), a Grant-inAid for scientific research (JSPS KAKENHI Grant number 25282141) from the Ministry of Education, Science, Sports, and Technology (MEXT), the Project for Development of Innovative Research on Cancer Therapeutics (P-DIRECT) and the Basic Science and Platform Technology Program for Innovative Biological Medicine from the Japan Agency for Medical Research and Development (AMED), and the Mochida Memorial Foundation for Medical and Pharmaceutical Research.

\section{References}

1. Burnett JC, Rossi JJ. RNA-based therapeutics: Current progress and future prospects. Chem Biol. 2012; 19:6071.

2. Sharma VK, Sharma RK, Singh SK. Antisense oligonucleotides: Modifications and clinical trials. Med Chem Comm. 2014; 5:1454-1471.

3. Wittrup A, Lieberman J. Knocking down disease: A progress report on siRNA therapeutics. Nat Rev Genet. 2015; 16:543-552.

4. Juliano RL, Carver K. Cellular uptake and intracellular trafficking of oligonucleotides. Adv Drug Deliv Rev. 2015; 87:35-45.

5. Raouane M, Desmaele D, Urbinati G, Massaad-Massade L, Couvreur P. Lipid conjugated oligonucleotides: A useful strategy for delivery. Bioconjug Chem. 2012; 23:1091-1104.

6. Nair JK, Willoughby JLS, Chan A, et al. Multivalent $\mathrm{N}$-acetylgalactosamine-conjugated siRNA localizes in hepatocytes and elicits robust RNAi-mediated gene silencing. J Am Chem Soc. 2014; 136:16958-16961.

7. Kataoka K, Harada A, Nagasaki Y. Block copolymer micelles for drug delivery: Design, characterization and biological significance. Adv Drug Deliv Rev. 2001; 47:113-131.

8. Kanasty R, Dorkin JR, Vegas A, Anderson D. Delivery materials for siRNA therapeutics. Nat Mater. 2013; 12:967-977.

9. Matsumura Y, Maeda H. A new concept for macromolecular therapeutics in cancer chemotherapy: Mechanism of tumoritropic accumulation of proteins and the antitumor agent Smancs. Cancer Res. 1986; 46:63876392.

10. Maeda H. Toward a full understanding of the EPR effect in primary and metastatic tumors as well as issues related to its heterogeneity. Adv Drug Deliv Rev. 2015; 91:3-6.

11. Davis ME, Chen Z, Shin DM. Nanoparticle therapeutics: An emerging treatment modality for cancer. Nat Rev Drug Discov. 2008; 7:771-782.

12. Duncan R, Vicent MJ. Polymer therapeutics-prospects for 21st century: The end of the beginning. Adv Drug Deliv Rev. 2013; 65:60-70.

13. Cabral H, Kataoka K. Progress of drug-loaded polymeric micelles into clinical studies. J Control Release. 2014; 190:465-476.

14. Miyata K, Nishiyama N, Kataoka K. Rational design of smart supramolecular assemblies for gene delivery: Chemical challenges in the creation of artificial viruses. Chem Soc Rev. 2012; 41:2562-2574.

15. Seymour LW, Duncan R, Strohalm J, Kopecek J. Effect of molecular weight of N-(2-hydroxypropyl) methacrylamide copolymers on body distribution and rate of excretion after subcutaneous, intraperitoneal, and intravenous administration to rats. J Biomed Mater Res. 1987; 21:1341-1358.

16. Choi HS, Liu W, Misra P, Tanaka E, Zimmer JP, Ipe BI, Bawendi MG, Frangioni JV. Renal clearance of quantum dots. Nat Biotechnol. 2007; 25:1165-1170.

17. Kano MR, Bae Y, Iwata C, Morishita Y, Yashiro M, Oka M, Fujii T, Komuro A, Kiyono K, Kaminishi M, Hirakawa K, Ouchi Y, Nishiyama N, Kataoka K, Miyazono K. Improvement of cancer-targeting therapy, using nanocarriers for intractable solid tumors by inhibition of TGF- $\beta$ signaling. Proc Natl Acad Sci U S A. 2007; 104:3460-3465.

18. Cabral H, Matsumoto Y, Mizuno K, Chen Q, Murakami M, Kimura M, Terada Y, Kano MR, Miyazono K, Uesaka M, Nishiyama N, Kataoka K. Accumulation of sub-100 $\mathrm{nm}$ polymeric micelles in poorly permeable tumours depends on size. Nat Nanotechnol. 2011; 6:815-823. 
19. Harada A, Kataoka K. Chain length recognition: Coreshell supramolecular assembly from oppositely charged block copolymers. Science. 1999; 283:65-67.

20. Hayashi K, Chaya H, Fukushima S, Watanabe S, Takemoto H, Osada K, Nishiyama N, Miyata K, Kataoka $\mathrm{K}$. Influence of RNA strand rigidity on polyion complex formation with block catiomers. Macromol Rapid Commun. 2016; 37:486-493.

21. Kataoka K, Togawa H, Harada A, Yasugi K, Matsumoto $\mathrm{T}$, Katayose S. Spontaneous formation of polyion complex micelles with narrow distribution from antisense oligonucleotide and cationic block copolymer in physiological saline. Macromolecules. 1996; 29:85568557.

22. Harada A, Togawa H, Kataoka K. Physicochemical properties and nuclease resistance of antisense oligodeoxynucleotides entrapped in the core of polyion complex micelles composed of poly(ethylene glycol)poly(L-lysine) block copolymers. Eur J Pham Sci. 2001; 13:35-42.

23. Tabernero J, Shapiro GI, LoRusso PM, et al. Firstin-humans trial of an RNA interference therapeutic targeting VEGF and KSP in cancer patients with liver involvement. Cancer Discov. 2013; 3:406-417.

24. Matsumoto S, Christie RJ, Nishiyama N, Miyata K, Ishii A, Oba M, Koyama H, Yamasaki Y, Kataoka K. Environment-responsive block copolymer micelles with a disulfide cross-linked core for enhanced siRNA delivery. Biomacromolecules. 2009; 10:119-127.

25. Kim HJ, Oba M, Pittella F, Nomoto T, Cabral H, Matsumoto Y, Miyata K, Nishiyama N, Kataoka K. PEGdetachable cationic polyaspartamide derivatives bearing stearoyl moieties for systemic siRNA delivery toward subcutaneous BxPC3 pancreatic tumor. J Drug Target. 2012; 20:33-42.

26. Kim HJ, Ishii $\mathrm{T}$, Zheng $\mathrm{M}$, Watanabe $\mathrm{S}$, Toh $\mathrm{K}$, Matsumoto Y, Nishiyama N, Miyata K, Kataoka K. Multifunctional polyion complex micelle featuring enhanced stability, targetability, and endosome escapability for systemic siRNA delivery to subcutaneous model of lung cancer. Drug Deliv Transl Res. 2014; 4:50-60.

27. Kim HJ, Miyata K, Nomoto T, Zheng M, Kim A, Liu X, Cabral H, Christie RJ, Nishiyama N, Kataoka K. siRNA delivery from triblock copolymer micelles with spatiallyordered compartments of PEG shell, siRNA-loaded intermediate layer, and hydrophobic core. Biomaterials 2014; 35:4548-4556.

28. Oe Y, Christie RJ, Naito M, Low SA, Fukushima S, Toh K, Miura Y, Matsumoto Y, Nishiyama N, Miyata $\mathrm{K}$, Kataoka K. Actively-targeted polyion complex micelles stabilized by cholesterol and disulfide crosslinking for systemic delivery of siRNA to solid tumors. Biomaterials. 2014; 35:7887-7895.

29. Gouda N, Miyata K, Christie RJ, Suma T, Kishimura A, Fukushima S, Nomoto T, Liu X, Nishiyama N, Kataoka K. Silica nanogelling of environment-responsive PEGylated polyplexes for enhanced stability and intracellular delivery of siRNA. Biomaterials 2013; 34:562-570.

30. Saito G, Swanson JA, Lee KD. Drug delivery strategy utilizing conjugation via reversible disulfide linkages: Role and site of cellular reducing activities. Adv Drug Deliv Rev. 2003; 55:199-215.

31. Kakizawa Y, Harada A, Kataoka K. Environment- sensitive stabilization of core-shell structured polyion complex micelle by reversible cross-linking of the core through disulfide bond. J Am Chem Soc. 1999; 121:11247-11248.

32. Kakizawa Y, Harada A, Kataoka K. Glutathione-sensitive stabilization of block copolymer micelles composed of antisense DNA and thiolated poly(ethylene glycol)block-poly(L-lysine): A potential carrier for systemic delivery of antisense DNA. Biomacromolecules. 2001; 2:491-497.

33. Christie RJ, Miyata K, Matsumoto Y, Nomoto T, Menasco D, Lai TC, Pennisi M, Osada K, Fukushima S, Nishiyama N, Yamasaki Y, Kataoka K. Effect of polymer structure on micelles formed between siRNA and cationic block copolymer comprising thiols and amidines. Biomacromolecules. 2011; 12:3174-3185.

34. Christie RJ, Matsumoto Y, Miyata K, Nomoto T, Fukushima S, Osada K, Halnaut J, Pittella F, Kim HJ, Nishiyama N, Kataoka K. Targeted polymeric micelles for siRNA treatment of experimental cancer by intravenous injection. ACS Nano. 2012; 6:5174-5189.

35. Tangsangasaksri M, Takemoto H, Naito M, Maeda Y, Sueyoshi D, Kim HJ, Miura Y, Ahn J, Azuma R, Nishiyama N, Miyata K, Kataoka K. siRNA-loaded polyion complex micelle decorated with chargeconversional polymer tuned to undergo stepwise response to intra-tumoral and intra-endosomal $\mathrm{pHs}$ for exerting enhanced RNAi efficacy. Biomacromolecules. 2016; 17:246-255.

36. Nishida H, Matsumoto Y, Kawana K, et al. Systemic delivery of siRNA by actively targeted polyion complex micelles for silencing the E6 and E7 human papillomavirus oncogenes. J Control Release. 2016; 231:29-37.

37. Pillay CS, Elliott E, Dennison C. Endolysosomal proteolysis and its regulation. Biochem J. 2002; 363:417429.

38. Knorr V, Russ V, Allmendinger L, Ogris M, Wagner E. Acetal linked oligoethylenimines for use as $\mathrm{pH}$-sensitive gene carriers. Bioconjug Chem. 2008; 19:1625-1634.

39. Anraku Y, Kishimura A, Kobayashi A, Oba M, Kataoka K. Size-controlled long-circulating PICsome as a ruler to measure critical cut-off disposition size into normal and tumor tissues. Chem Commun. 2011; 47:6054-6056.

40. Naito M, Ishii T, Matsumoto A, Miyata K, Miyahara Y, Kataoka K. A phenylboronate-functionalized polyion complex micelle for ATP-triggered release of siRNA. Angew Chem Int Ed Engl. 2012; 51:10751-10755.

41. Traut TW. Physiological concentrations of purines and pyrimidines. Mol Cell Biochem. 1994; 140:1-22.

42. Jeong JH, Kim SW, Park TG. Molecular design of functional polymers for gene therapy. Prog Polym Sci. 2007; 32:1239-1274.

43. Wagner E, Cotten M, Foisner R, Birnstiel ML. Transferrin-polycation-DNA complexes: The effect of polycations on the structure of the complex and DNA delivery to cells. Proc Natl Acad Sci U S A. 1991; 88:4255-4259.

44. Davis ME, Zuckerman JE, Choi CHJ, Seligson D, Tolcher A, Alabi CA, Yen Y, Heidel JD, Ribas A. Evidence of RNAi in humans from systemically administered siRNA via targeted nanoparticles. Nature. 2010; 464:1067-1070.

45. Wiley DT, Webster P, Gale A, Davis ME. Transcytosis and brain uptake of transferrin-containing nanoparticles 
by tuning avidity to transferrin receptor. Proc Natl Acad Sci U S A. 2013; 110:8662-8667.

46. Kumar P, Wu H, McBride JL, Jung KE, Kim MH, Davidson BL, Lee SK, Shankar P, Manjunath N. Transvascular delivery of small interfering RNA to the central nervous system. Nature. 2007; 448:39-43.

47. Arap W, Pasqualini R, Ruoslahti E. Cancer treatment by targeted drug delivery to tumor vasculature in a mouse model. Science. 1998; 279:377-380.

48. Schiffelers RM, Ansari A, Xu J, Zhou Q, Tang Q, Storm G, Molema G, Lu PY, Scaria PV, Woodle MC. Cancer siRNA therapy by tumor selective delivery with ligandtargeted sterically stabilized nanoparticle. Nucleic Acids Res. 2004; 32:e149.

49. Shayakhmetov DM, Eberly AM, Li ZY, Lieber A. Deletion of penton RGD motifs affects the efficiency of both the internalization and the endosome escape of viral particles containing adenovirus serotype 5 or 35 fiber knobs. J Virol. 2005; 79:1053-1061.

50. Ishii T, Miyata K, Anraku Y, Naito M, Yi Y, Jinbo T, Takae S, Fukusato Y, Hori M, Osada K, Kataoka K. Enhanced target recognition of nanoparticles by cocktail PEGylation with chains of varying lengths. Chem Commun. 2016; 52:1517-1519.

51. Smits RG, Koper GJM, Mandel M. The influence of nearest- and next-nearest-neighbor interactions on the potentiometric titration of linear poly(ethylenimine). J Phys Chem. 1993; 97:5745-5751.

52. Boussif O, Lezoualch F, Zanta MA, Mergny MD, Scherman D, Demeneix B, Behr JP. A versatile vector for gene and oligonucleotide transfer into cells in culture and in-vivo - polyethylenimine. Proc Natl Acad Sci U S A. 1995; 92:7297-7301.

53. Neu M, Fischer D, Kissel T. Recent advances in rational gene transfer vector design based on poly(ethylene imine) and its derivatives. J Gene Med. 2005; 7:992-1009.

54. Fischer D, Li Y, Ahlemeyer B, Krieglstein J, Kissel T. In vitro cytotoxicity testing of polycations: Influence of polymer structure on cell viability and hemolysis. Biomaterials. 2003; 24:1121-1131.

55. Moghimi SM, Symonds P, Murray JC, Hunter AC, Debska G, Szewczyk A. A two-stage poly(ethylenimine)mediated cytotoxicity: Implications for gene transfer/ therapy. Mol Ther. 2005; 11:990-995.

56. Kim YH, Park JH, Lee M, Kim YH, Park TG, Kim SW. Polyethylenimine with acid-labile linkages as a biodegradable gene carrier. J Control Release. 2005; 103:209-219.

57. Lee Y, Mo H, Koo H, Park JY, Cho MY, Jin GW, Park JS. Visualization of the degradation of a disulfide polymer, linear poly(ethylenimine sulfide), for gene delivery. Bioconjug Chem. 2007; 18:13-18.

58. Miyata K, Christie RJ, Suma T, Takemoto H, Uchida H, Nishiyama N, Kataoka K. Fine-tuning of repeating aminoethylene units in poly(aspartamide) side chains for enhanced siRNA delivery. In: Tailored Polymer Architectures for Pharmaceutical and Biomedical Applications (Scholz C, Kressler J, eds.). American Chemical Society, USA, 2013; pp. 189-196.

59. Miyata K, Oba M, Nakanishi M, Fukushima S, Yamasaki Y, Koyama H, Nishiyama N, Kataoka K. Polyplexes from poly(aspartamide) bearing 1,2-diaminoethane side chains induce $\mathrm{pH}$-selective, endosomal membrane destabilization with amplified transfection and negligible cytotoxicity. J Am Chem Soc. 2008; 130:16287-16294.

60. Walker GF, Fella C, Pelisek J, Fahrmeir J, Boeckle $\mathrm{S}$, Ogris M, Wagner E. Toward synthetic viruses: Endosomal pH-triggered deshielding of targeted polyplexes greatly enhances gene transfer in vitro and in vivo. Mol Ther. 2005; 11:418-425.

61. Takae S, Miyata K, Oba M, Ishii T, Nishiyama N, Itaka K, Yamasaki Y, Koyama H, Kataoka K. PEG-detachable polyplex micelles based on disulfide-linked block catiomers as bioresponsive nonviral gene vectors. J Am Chem Soc. 2008; 130:6001-6009.

62. Rozema DB, Ekena K, Lewis DL, Loomis AG, Wolff JA. Endosomolysis by masking of a membrane-active agent (EMMA) for cytoplasmic release of macromolecules. Bioconjug Chem. 2003; 14:51-57.

63. Lee Y, Miyata K, Oba M, Ishii T, Fukushima S, Han M, Koyama H, Nishiyama N, Kataoka K. Chargeconversion ternary polyplex with endosome disruption moiety: A technique for efficient and safe gene delivery. Angew Chem Int Ed Engl. 2008; 47:5163-5166.

64. Maeda Y, Pittella F, Nomot T, Takemoto H, Nishiyama N, Miyata K, Kataoka K. Fine-tuning of charge-conversion polymer structure for efficient endosomal escape of siRNA-loaded calcium phosphate hybrid micelles. Macromol Rapid Commun. 2014; 35:1211-1215.

(Received September 14, 2016; Revised September 28, 2016; Accepted October 7, 2016) 